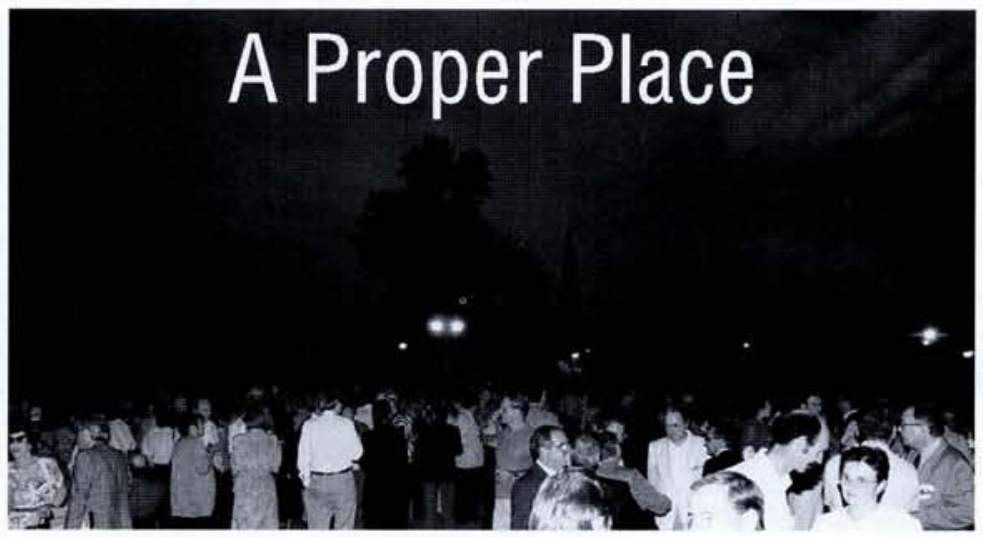

The 10th EPS General Conference Trends in Physics, organized by the Portuguese Physical Society and The Royal Spanish Physical Society, was held in Sevilla on 9 - 13 September 1996. This issue of Europhysics News contains reports of several of the technical symposia, the Cecil Powell Memorial Lecture and an item on physics eduction in Europe presented at a symposium on physics and education. Highlights from other symposia will be published in the next issue of Europhysics News, together with a summary of the plenary talks and reports of symposia devoted to employment opportunities for young physicists and university - industry relations.



C. Matos Ferreira, who co-chaired the conference, making his closing address. Seated are (from left to right) , R. Conde, a City Councillor who represented the Mayor of Sevilla, H. Walther who chaired the International Programme Committee, H. Schopper, the EPS President, and A. Troncoso, Director of the University of Sevilla's Research Secretariat, who represented the University's Rector.
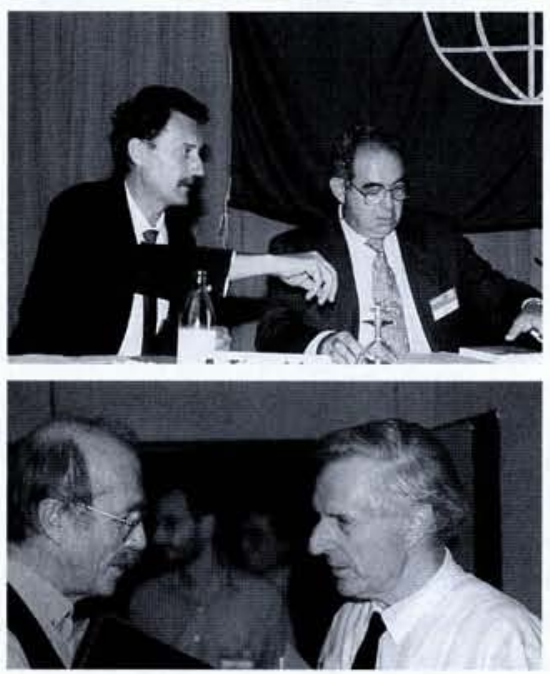

M. Lozano Leyva, on the left, with A. Tiemblo Ramos, a Past-President of The Royal Spanish Physical Society, who opened the plenary lectures with a talk entitled The road to the question modelled on the Santiago road. He stressed the need to recover a role for science while recognizing that science is too important to be left either to scientists or to people in general.

Sir Arnold Wolfendale (on the right), President of the UK's Institute of Physics, who gave the Cecil Powell Memorial Lecture entitled Public Understanding of Physics - The British Experience (see page 168). He is seen here with $M$. Schwoerer, the President of the German Physical Society.



Entertaining distinguished speakers. Seen in the front row are K. von Klitzing (second from left) and R.L. Mössbauer (third from left). N.F. Ramsey is seen standing at the back in the middle, with M. Perl on the right, who closed the conference with a talk on Conventional and speculative experiments in elementary particle physics.
SEVILLA There could not be a more appropriate city to host an EPS General Conference, for as the President pointed out in his opening address, Sevilla harbours a tradition of international contacts, an exploratory spirit and an engagement to push future developments - features that physics also strives to acquire. And the city lives up to the challenges of our times. For it not only founded one of Europe's oldest universities but also hosted a recent World Exhibition. Indeed, Seville has become a symbol for the symbiosis of different cultures, as witnessed by a remarkable collection of unique buildings reaching back to the time the city was the capital of the Moorish Almohaden kingdom, and spanning the period when it became Spain's most important port following the discovery of the Americas.

Recalling the origins of a nation's cultural heritage is appropriate because the relation between science and society remains uneasy in spite of science's major contribution to economic progress. Governments and the public question whether science can help solve pressing short-term problems. What is often forgotten is that the basis for our well-being was laid down some time ago by scientists, and in particular physicists, who were largely motivated by curiosity and not the rewards of the market place. To maintain momentum, the President singled out the need to reinforce multidisciplinarity within the unity of physics. Concepts developed in a given area fertilise other areas: this is one of the great strengths of physics. It should become the basis for an intensified multidisciplinary effort, aided by meetings such as the EPS General Conference, which has as its main purpose the dissemination of information and ideas.

\section{A Message from the Organizers}

During the 9 th General Conference of the European Physical Society held in Florence in 1993, the Executive Committee decided to accept an offer from the Portuguese Physical Society and The Royal Spanish Physical Society to organize the 1oth event. The place proposed by both societies was Sevilla, with Carlos M. Ferreira, from the University of Lisbon, and Manuel Lozano Leyva, from the University of Sevilla, chairing the conference. The International Programme Committee chaired by Herbert Walther from the University of Munich then set to work. It elaborated a extremely interesting programme that was divided into 20 plenary talks and 18 symposia.

The selection of subjects, speakers and symposia chairs attracted around 530 participants in total. The organizers found support from various local, regional, national, European and international institutions. The University of Sevilla, the Andalusian Government, the Spanish Ministry of Science and Education, and the European Commission contributed in an major way towards covering the cost of the conference. The participation of more than 30 physicists from east and central Europe was made possible thanks to financial support from NATO in the form of grants.

Participants enjoyed not only an outstanding scientific programme but also the splendid atmosphere and surroundings offered by the city of Sevilla. During the conference, the Executive Committee decided to hold the 11th EPS General Conference in London on 6-10 September 1999. Our best wishes to the organizers.

M. Lozano Leyva 